\title{
Setting Priors and Enforcing Constraints on Matches for Nonlinear Registration of Meshes
}

\author{
Benoît Combès and Sylvain Prima \\ INSERM, U746, F-35042 Rennes, France \\ INRIA, VisAGeS Project-Team, F-35042 Rennes, France \\ University of Rennes I, CNRS, UMR 6074, IRISA, F-35042 Rennes, France \\ \{bcombes, sprima\}@irisa.fr \\ http://www.irisa.fr/visages
}

\begin{abstract}
We show that a simple probabilistic modelling of the registration problem for surfaces allows to solve it by using standard clustering techniques. In this framework, point-to-point correspondences are hypothesized between the two free-form surfaces, and we show how to specify priors and to enforce global constraints on these matches with only minor changes to the optimisation algorithm. The purpose of these two modifications is to increase its capture range and to obtain more realistic geometrical transformations between the surfaces. We conclude with some validation experiments and results on synthetic and real data.
\end{abstract}

\section{Introduction}

In medical image analysis, nonlinear registration is a key tool to study the normal and abnormal anatomy of body structures. It allows to spatially normalise different subjects in a common template, to build the average anatomy in a population and to assess the variance about this average, and ultimately to perform group studies via statistical analyses. Many methods have been dedicated to deal with grey level volumes directly, while others have been devised to tackle surfaces representing anatomical structures (e.g. after segmentation from MRI or CT) 1]. The last approach allows a more focused analysis of structures of interest, and is the topic of our paper. In Section 2 we show that a simple probabilistic modelling of the registration problem allows to solve it by using standard clustering techniques. In this framework, point-to-point correspondences are hypothesized between the two free-form surfaces, and we show how to specify priors (Section 3) and to enforce global constraints (Section 4) on these matches with only minor changes to the optimisation algorithm. This extends our previous work on the same problem [2]. The purpose of these two modifications is to increase its capture range and to obtain more realistic geometrical transformations between the surfaces. We conclude with some validation experiments and results on synthetic and real data (Section 5 ). 


\section{Surface Registration as a Clustering Problem}

The problem of interest in this paper is to find the transformation $T$ best superposing two free-form surfaces $X$ and $Y$ (represented by point clouds or meshes). A convenient probabilistic viewpoint on this classical problem is to consider the surface $Y$ as a noised version of $T(X)$. If we note $Y=\left(y_{j}\right)_{j=1 \ldots \operatorname{card}(Y)}$ and $X=\left(x_{k}\right)_{k=1 \ldots \operatorname{card}(X)}$, and if we hypothesize an isotropic Gaussian noise, a simple way to formulate this viewpoint is to assume that each sample $y_{j}$ has been drawn independently from any one of $\operatorname{card}(X)$ possible 3-variate normal distributions with means $T\left(x_{k}\right)$ and covariance matrices $\sigma^{2} I$ (with $\sigma$ unknown).

This way, the registration problem becomes a clustering problem, whose challenge is i) to find the label of each point $y_{j}$, i.e. the one out of $\operatorname{card}(X)$ possible distributions from which $y_{j}$ has been drawn, and ii) to estimate the parameters of these $\operatorname{card}(X)$ distributions. The connection between registration and clustering becomes clear when one realises that i) actually amounts to match each point $y_{j}$ in $Y$ with a point $x_{k}$ in $X$, while ii) simply consists in computing $T$ given these matches. This viewpoint is extremely fruitful, as it allows one to refer to classical clustering techniques and especially the maximum likelihood principle to solve the registration problem. Two different paradigms have been especially followed in this context [3]. Let us introduce some notations first:

$$
\begin{aligned}
& \forall k \in 1 \ldots \operatorname{card}(X), p_{k}(. ; T)=\mathcal{N}\left(T\left(x_{k}\right), \sigma^{2} I\right) \\
& \forall j \in 1 \ldots \operatorname{card}(Y), \forall k \in 1 \ldots \operatorname{card}(X), z_{j k}=1 \text { if } y_{j} \text { comes from } p_{k}(. ; T), 0 \text { else }
\end{aligned}
$$

In the Classification ML (CML) approach, one tries to find the indicator variables $z_{j k}$ and the parameter $T$ so as to maximise the criterion $C L$ [4]:

$$
C L=\prod_{y_{j} \in Y} \prod_{x_{k} \in X}\left[p_{k}\left(y_{j} ; T\right)\right]^{z_{j k}}
$$

The problem is typically solved by the Classification EM (CEM) algorithm [5], which can be shown to find an at least local maximum of the criterion $C L$ and proceeds as follows, in an iterative way, starting from an initial value $\tilde{T}$ :

EC-step: $\forall j, \tilde{z}_{j k}=1$ if $k$ maximises $p_{k}\left(y_{j} ; \tilde{T}\right), 0$ else M-step: $\tilde{T}=\arg \min _{T} \sum_{j k} \tilde{z}_{j k}\left\|y_{j}-T\left(x_{k}\right)\right\|^{2}$

In other words, the Expectation-Classification (EC) step consists in matching each point $y_{j}$ of $Y$ with the closest point in $\tilde{T}(X)$, while the Maximisation (M) step consists in computing the transformation best superposing these pairs of matched points. In case $T$ is a rigid-body transformation, this is nothing else than the popular ICP algorithm [6].

In the ML approach, the indicator values $z_{j k}$ are no longer considered as unknown quantities to estimate, but rather as hidden/unobservable variables of the problem. This is actually a drastic and fundamental change of viewpoint, as the focus is no longer on assigning each $y_{j}$ to one of the distributions $p_{k}$ 
but rather on estimating the parameters of the Gaussian mixture made of these distributions. If we involve priors $\pi_{j k}$ on the indicator variables $\left(\forall j, k, 0<\pi_{j k}<\right.$ 1 , and $\forall j, \sum_{k} \pi_{j k}=1$ ), the likelihood then simply writes [7]:

$$
L=\prod_{y_{j} \in Y} \sum_{x_{k} \in X} \pi_{j k} p_{k}\left(y_{j} ; T\right)
$$

In essence, the prior $\pi_{j k}$ conveys the probability that the point $y_{j}$ comes from the distribution $p_{k}$ without knowing anything else. The criterion $L$ can be maximised by using the popular EM algorithm, which converges to an at least local maximum of the likelihood [8]. If we consider the priors $\pi_{j k}$ as known beforehand and if we introduce the notation $\gamma_{j k}$ as the posterior probability of the hidden indicator variable $z_{j k}$ to be equal to 1 , the EM algorithm writes:

$\mid \begin{aligned} & \text { E-step: } \tilde{\gamma}_{j k}=\frac{\pi_{j k} \exp \left[-\left\|y_{j}-\tilde{T}\left(x_{k}\right)\right\|^{2} /\left(2 \sigma^{2}\right)\right]}{\sum_{i} \pi_{j i} \exp \left[-\left\|y_{j}-\tilde{T}\left(x_{i}\right)\right\|^{2} /\left(2 \sigma^{2}\right)\right]} \\ & \text { M-step: } \tilde{T}=\arg \min _{T} \sum_{j k} \tilde{\gamma} \tilde{\gamma}_{j k}\left\|y_{j}-T\left(x_{k}\right)\right\|^{2}\end{aligned}$

To our knowledge, Granger \& Pennec [9] were the first to formulate the problem this way, and they proposed the so-called EM-ICP as a simplified version of the previous algorithm for rigid-body registration, where the priors $\pi_{j k}$ were considered as uniform. They noted that the parameter $\sigma$, which is not estimated in this framework, acts as a scale parameter, and that it can be given an initial value and decreased throughout the iterations for improved performances.

Interpretation \& Extensions. Intuitively, the EM approach is a fuzzy version of the CEM. It appears clearly from the iterative formulas of both algorithms that the classification likelihood is an "all-or-nothing" version of the likelihood, leading to a "bumpier" and harder-to-maximise criterion, something that is well known by those who are familiar with the ICP algorithm. Note that the ML formulation and the EM algorithm lead to the same iterative formulas that would have resulted from the addition of a barrier function on the indicator variables in the ICP criterion [10. This formalism is not limited to rigid-body transformations, and can be easily used for any $T$, the challenge being to choose $T$ such that the M-step remains tractable. In particular, the ML estimation can be easily turned into a MAP problem with only slight modifications to the optimisation scheme, as shown by Green [1]. This allows to view $T$ as a random variable, on which priors (acting as regularisers on $T$ ) can be easily specified. Different choices have been proposed for $T$ and associated priors in this context, such as the thin plate splines [10] or locally affine transformations [12]. If $p(T)$ is a prior of the form $p(T) \propto \exp (-\alpha R(T))$ then the optimal transformation can be found using the MAP principle (also termed penalised ML) and the EM algorithm with only a slight modification to the M-step (addition of $\alpha R(T))$. 


\section{Setting Priors on Matches}

\subsection{Why Using Priors on Matches?}

Most works in this context have been focused on designing transformations and related priors allowing to i) compute realistic deformations between the two surfaces and ii) keep the M-step tractable. Much little has been done to enforce similar constraints on the matches (E-step). Dropping the priors $\pi_{j k}$, as done in the ICP of Besl \& McKay or the EM-ICP of Granger \& Pennec amounts to say that the posteriors $\gamma_{j k}$ are only estimated using the spatial distance between the points $y_{j}$ and $T\left(x_{k}\right)$ (E-step). This is unsatisfactory, for two reasons. First, this distance is highly conditioned by the previous estimation of $T$, which in turn depends on the previous estimation of $\gamma_{j k}$ and so on. This chicken-and-egg problem limits the capture range of the algorithm, which is likely to converge to a bad solution if no good initial $T$ is given. Second, in medical imaging it is difficult to design a physical model $T$ capturing the expected deformation between two structures. Thus the global maximiser of the ML criterion is likely not to be realistic. By specifying relevant priors $\pi_{j k}$, we provide a way to partially alleviate these two limitations. First, it allows to introduce additional information on matches independent of the transformation and thus to compute reliable posteriors even for a bad initial estimate of $T$. Second, it allows to modify the criterion in a way that its global maximiser is a more realistic transformation.

\subsection{Building Priors on Matches}

In this section we show how to design the priors $\pi_{j k}$ to encode very heterogeneous types of a priori knowledge on the matches, such as "two points with similar curvatures are more likely to be matched than others" as well as knowledge of the labels of structures in the objects to be matched (e.g. gyri/sulci in cortical registration). In practice, we choose to design $\pi=\left(\pi_{j k}\right)$ such that $\pi_{j k} \propto \exp \left(-\beta c\left(y_{j}, x_{k}\right)\right)$ where $c: X \times Y \rightarrow \mathbb{R}^{+}$conveys the cost of matching points $y_{j}$ and $x_{k}$, independently of $T$. The parameter $\beta>0$ weighs the influence of $\pi_{j k}$ over $\left\|y_{j}-T\left(x_{k}\right)\right\|$ during the E-step. Depending on the information to encode (continuous value or label), we propose two approaches to build $c$.

Using descriptors. $c$ can be computed via the comparison between continuous values (or vectors) $d(x)$ describing the surface around the considered points. To account for potential inaccuracies on $d($.$) , we define the measure as: c^{d}\left(y_{j}, x_{k}\right)=$ 0 if $\left\|d\left(y_{j}\right)-d\left(x_{k}\right)\right\|<\tau ;=$ penalty $>0$ else. To the best of our knowledge, there exists no descriptor invariant to any nonlinear transformation. However, one can use some descriptors invariant to more constrained transformations (Fig. 1, left):

- the shape index $\operatorname{sh}(x)[13$ that describes the local shape irrespective of the scale and that is invariant to similarities

- the curvedness $c u(x)[13$ that specifies the amount of curvature and that is invariant to rigid-body transformations

- the (normalised) total geodesic distance $\operatorname{tgd}(x)$ [14 that is invariant to isometries in the shape space (including non-elastic deformations). 


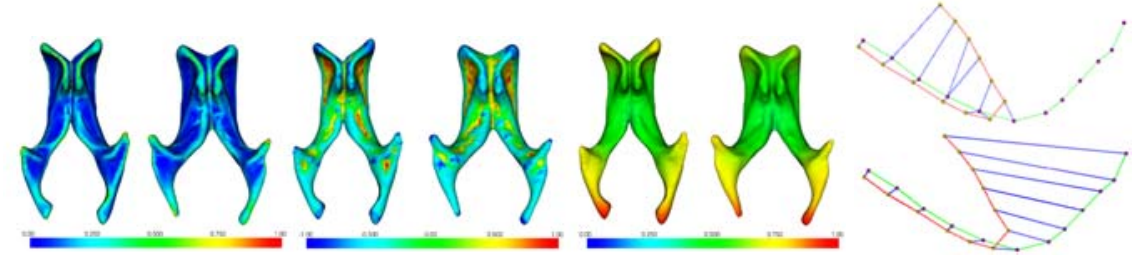

Fig. 1. Left: Mapping of descriptor values: From left to right: curvedness, shape index and total geodesic distance on two lateral ventricles. Homologous anatomical landmarks yield qualitatively the same descriptor values. Right: point-based matching (top) vs line-based matching performed by our algorithm (bottom).

Using labels. $c$ can be computed via the comparison between labels on points (cortical sulci/gyri). We define: $c\left(y_{j}, x_{k}\right)=0$ if points $j$ and $k$ have compatible labels; = penalty $>0$ else. In practice, we extract the crest lines from both meshes as they constitute salient features. Each point is given a label depending on whether it belongs to a crest line or not. Then, we define $c_{\text {crest }}\left(y_{j}, x_{k}\right)=0$ if $y_{j}$ and $x_{k}$ have the same label and $c\left(y_{j}, x_{k}\right)=$ penalty else.

Mixing the two approaches. In practice, we choose to mix the previous four sources of information to build the function $c: c\left(y_{j}, x_{k}\right)=a_{1} c^{s h}\left(y_{j}, x_{k}\right)+$ $a_{2} c^{c u}\left(y_{j}, x_{k}\right)+a_{3} c^{t g d}\left(y_{j}, x_{k}\right)+a_{4} c_{\text {crest }}\left(y_{j}, x_{k}\right)$ with $a_{1}+a_{2}+a_{3}+a_{4}=1$. Parameters $a_{i}$ allow to weigh the different terms. Their values is application-dependent and will not be studied in this paper, in which we set them to 0.25 .

\subsection{Efficient Implementation}

In practice, we do not consider all points during the computation of $\gamma_{j k}$ (E-step). For that, we consider $p_{k}(. ; T)$ as a truncated Gaussian pdf with cut-off distance $\delta$. This allows to reduce the computational burden (by the use of a $k \mathrm{~d}$-tree) and increase robustness. It can be shown that our algorithm still converges to an at least local maximum of the new (truncated) criterion. The E-Step becomes:

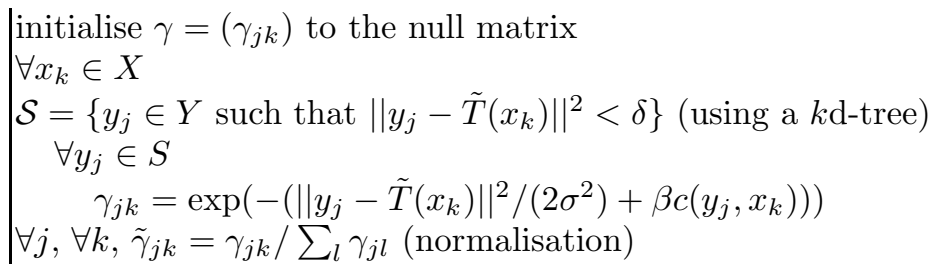

Moreover, we choose to initialise $\alpha$ (regularisation weight), $\sigma$ (scale parameter) and $\beta$ (prior weight) with high values and reduce them throughout iterations. 


\section{Enforcing Global Constraints on Matches}

In the formalism presented so far, the matches in the E-step are performed on an individual basis without any global constraint such as one-to-one matches for instance. Another desirable global constraint is that some geometrical relationships between points in $Y$ should be preserved between their correspondences in $X$. Thus if we consider each crest line in $Y$ as a set of ordered points, then their correspondences must i) lie on the same crest line in $X$ and ii) be ordered in the same way (up to the orientation of the line) (Fig. 1 right). To enforce these two constraints, let us introduce some notations first. Let $L$ and $M$ be the sets of crest lines of $Y$ and $X$, each crest line being defined as a set of ordered points. Let $u=\left(u^{l m}\right)$ be a block matrix whose lines (resp. columns) correspond to the concatenated crest lines of $Y(\operatorname{resp} . X)$. Then $u_{j k}^{l m}$ is the indicator variable $u_{j k}^{l m}=1$ iff $y_{j}$ in crest line $l$ corresponds to the point $x_{k}$ in crest line $m$. The two constraints i) and ii) are specified as follows: the submatrix $u^{l m}$ is either null (the line $l$ does not match with the line $m$ ) or contains one 1 per line, with the $1 \mathrm{~s}$ drawing a "staircase" going to the left or to the right, all "steps" of the staircase having potentially different widths and different heights. Then we propose to maximise the following criterion over $T$ and $u$ having this staircase structure:

$$
L-C L=\left[\prod_{y_{j} \in Y \backslash L} \sum_{x_{k} \in X \backslash M} \pi_{j k} p_{k}\left(y_{j} \mid T\right) \times \prod_{l \in L} \prod_{y_{j} \in l} \sum_{x_{k} \in m}\left[p_{k}\left(y_{j} \mid T\right)\right]^{u_{j k}^{l m}}\right] \times p(T)
$$

This criterion is an hybrid between the classification likelihood and the likelihood approaches. Introducing $u$ only modifies the E-step of the algorithm, in which $u$ and $\gamma$ can be estimated independently. The algorithm becomes:

E-Step: compute $\tilde{\gamma}$ as before $\forall l$, compute $\tilde{u}^{l m}$ respecting the staircase structure and maximising $\prod_{y_{j} \in l} \sum_{x_{k} \in m}\left[p_{k}\left(y_{j} \mid \tilde{T}\right)^{\tilde{u}_{j k}^{l m}}\right]$

M-Step: $\tilde{T}=\arg \min _{T} \sum_{y_{j} \in Y \backslash L, x_{k} \in X \backslash M} \tilde{\gamma}_{j k}\left\|y_{j}-T\left(x_{k}\right)\right\|^{2}$ $+\sum_{l \in L} \sum_{y_{j} \in l, x_{k} \in m} \tilde{u}_{j k}^{l m}\left\|y_{j}-T\left(x_{k}\right)\right\|^{2}+\alpha R(T)$

To our knowledge, an exhaustive search is the only way to maximise the proposed criterion over $u$. Instead, we propose to design an heuristic algorithm to do so, that extends the one proposed by Subsol [15] and consists of two steps: i) finding the crest line $m \in M$ that corresponds to each $l \in L$ and ii) starting from different initial potential matches and assigning iteratively each point $y_{j} \in l$ to a point $x_{k} \in m$ by keeping the staircase structure of the submatrix $u^{l m}$.

\section{$5 \quad$ Experiments and Results}

In the following we choose to adopt a locally affine regularisation [12] (with only the translational part) because of its ability to perform efficiently $(\sim 7 \mathrm{~min})$ on large datasets $(\sim 50 \mathrm{~K}$ points $)$. 


\subsection{Experiments on Synthetic Data}

Generation of ground truth data. We first segment a structure $X$ (typically, a pair of lateral ventricles or caudate nuclei, giving surfaces of about $10 \mathrm{~K}$ points, itksnap.org) from a 3T T1-weighted brain MRI of a healthy subject. Then $Y$ is generated from $X$ by applying a random thin plate spline transformation [2]. Then, we add a uniform Gaussian noise of std $0.5 \mathrm{~mm}$ on each point of the deformed surface and remove groups of adjacent vertices to generate holes. This way we generate ground truth pairs of 100 ventricles and 100 caudate nuclei.

Evaluation. We evaluate the error by computing the mean distance between homologous points after registration using different strategies. The results are reported in Tab. 1 and an example is displayed in Fig. 2. They show the strong added value of using priors. The error is further reduced when using constraints, which ensure that the ordering of the points on the lines is kept unchanged, and thus help the algorithm to obtain anatomically coherent matches elsewhere.

Table 1. Experiments on synthetic data (stats). Mean and std ( $\mathrm{mm}$ ) of the registration error for the 100 ventricles and 100 caudate nuclei by varying the parameters.

\begin{tabular}{|c|c|c|c|}
\hline & no prior/no constraint & prior/no constraint & prior/constraint \\
\hline ventricle & $2.19 \pm 0.82$ & $1.43 \pm 0.91$ & $1.39 \pm 0.93$ \\
\hline caudate nuclei & $1.54 \pm 0.43$ & $1.04 \pm 0.57$ & $0.98 \pm 0.56$ \\
\hline
\end{tabular}

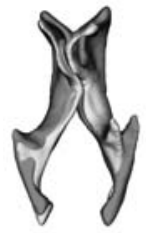

a

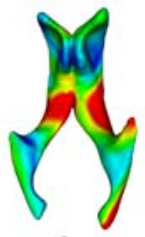

$\mathbf{b}$

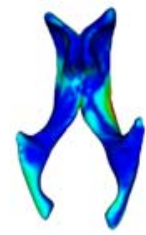

c

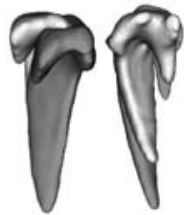

d

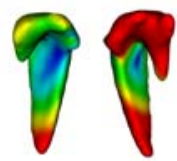

e

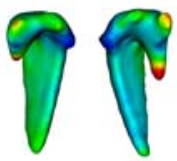

f

Fig. 2. Experiments on synthetic data. From left to right: Experiments on two different structures: ventricles and caudate nuclei. a) and d) original and deformed; b) and e) mapping of registration error ( $\mathrm{mm}$ ) without using prior/constraint; c) and f) mapping of registration error using prior/constraint.

\subsection{Experiments on Real Data}

In a first experiment, we choose $X$ and $Y$ as two different lateral ventricles and manually extract a set of 8 anatomical landmarks common to $X$ and $Y$. We then apply a random affine transformation $A$ to $Y$, register $A(Y)$ to $X$ with and without using priors/constraints, and evaluate the registration error on landmarks. We perform this experiment 100 times (but we display only 10 on Fig. 3 for better clarity). We observe a mean error of $1.73 \pm 1.24 \mathrm{~mm}$ with the priors/constraints 


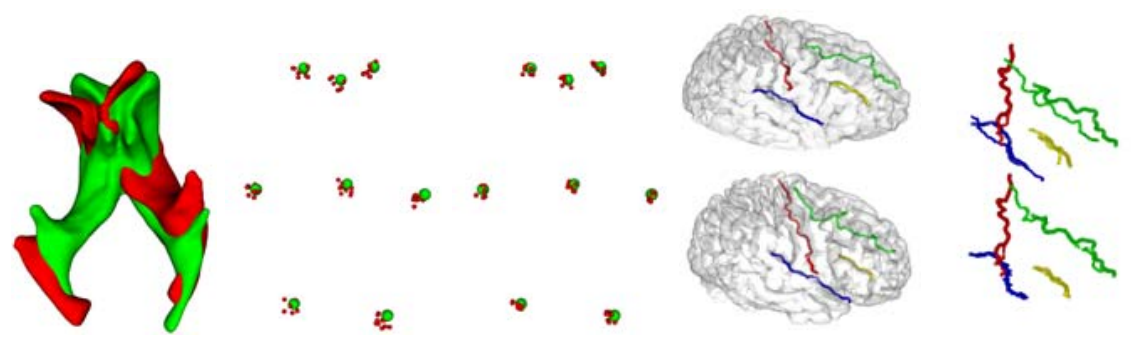

Fig. 3. Experiments on real data. Ventricles: From left to right : $X$ (green) and $A(Y)$ (red) before registration, position of the 8 anatomical landmarks of the 10 random experiments after registration without priors/constraints and with priors/constraints. Brain: From left to right and top to bottom: 1) brain 1 (top) and brain 2 (bottom); 2) brain 2 (with sulci shown in transparency) towards brain 1 without (top) and with (bottom) using priors/constraints. The four sulci are the central (red), lateral (blue), superior frontal (green) and inferior frontal (yellow) sulci.

and $2.55 \pm 2.46 \mathrm{~mm}$ without (Fig. 3, left). In a second experiment, we segment the brain from T1-weighted MRI data of two healthy subjects (300,000 points, brainvisa.info), and we extract four sulcal fundus beds (using our algorithm for crest lines) and label them manually for each subject. Then we register the two surfaces with and without using priors/constraints. The distance between the homologous sulci after registration is used as a quality metric. It is evaluated to be $3.3 \mathrm{~mm}$ in the first case and $6.8 \mathrm{~mm}$ in the second (Fig. 3, right).

\section{Conclusion and Perspectives}

We proposed techniques to set priors and enforce some global geometrical constraints on matches for ML-based nonlinear registration of surfaces. The priors on matches give a flexible way to devise structure-specific registration algorithms. They ideally complement the global, generic, prior on the transformation and thus help to obtain a result coherent with the application of interest. In addition, they provide a convenient framework to include additional knowledge (segmentation, landmarks, etc.) provided by experts when available. In the future, comparisons with other recent methods, especially landmark-free approaches [16 and others that do not resort to point-to-point correspondences [17] will be led.

\section{References}

1. Audette, M.A., Ferrie, F.P., Peters, T.M.: An Algorithmic Overview of Surface Registration Techniques for Medical Imaging. MIA 4, 201-217 (2000)

2. Combès, B., Prima, S.: Prior Affinity Measures on Matches for ICP-Like Nonlinear Registration of Free-Form Surfaces. IEEE ISBI (2009)

3. Marriott, F.H.C.: Separating Mixtures of Normal Distributions. Biometrics 31(3), 767-769 (1975) 
4. Scott, A.J., Symons, M.J.: Clustering Methods Based on Likelihood Ratio Criteria. Biometrics 27(2), 387-397 (1971)

5. Celeux, G., Govaert, G.: A Classification EM Algorithm for Clustering and Two Stochastic Versions. Computational Statistics and Data Analysis 14(3), 315-332 (1992)

6. Besl, P., McKay, N.: A Method for Registration of 3-D Shapes. IEEE PAMI 14(2), 239-256 (1992)

7. Day, N.E.: Estimating the Components of a Mixture of Normal Distributions. Biometrika 56(3), 463-474 (1969)

8. Dempster, A.P., Laird, N.M., Rubin, D.B.: Maximum Likelihood from Incomplete Data via the EM Algorithm. Journal of the Royal Statistical Society. Series B (Methodological) 39(1), 1-38 (1977)

9. Granger, S., Pennec, X.: Multi-scale EM-ICP: A fast and robust approach for surface registration. In: Heyden, A., Sparr, G., Nielsen, M., Johansen, P. (eds.) ECCV 2002. LNCS, vol. 2353, pp. 418-432. Springer, Heidelberg (2002)

10. Chui, H., Rangarajan, A.: A Feature Registration Framework Using Mixture Models. In: IEEE MMBIA, pp. 190-197 (2000)

11. Green, P.J.: On Use of the EM for Penalized Likelihood Estimation. Journal of the Royal Statistical Society. Series B (Methodological) 52(3), 443-452 (1990)

12. Combès, B., Prima, S.: New algorithms to map asymmetries of 3D surfaces. In: Metaxas, D., Axel, L., Fichtinger, G., Székely, G. (eds.) MICCAI 2008, Part I. LNCS, vol. 5241, pp. 17-25. Springer, Heidelberg (2008)

13. Koenderink, J.J., van Doorn, A.J.: Surface Shape and Curvature Scales. Image and Vision Computing 10(8), 557-564 (1992)

14. Aouada, D., Feng, S., Krim, H.: Statistical Analysis of the Global Geodesic Function for 3D Object Classification. In: IEEE ICASSP, pp. I-645-I-648 (2007)

15. Subsol, G., Thirion, J.-P., Ayache, N.: A General Scheme for Automatically Building 3D Morphometric Anatomical Atlases: application to a Skull Atlas. MIA 2, 37-60 (1998)

16. Yeo, B.T.T., Sabuncu, M.R., Vercauteren, T., Ayache, N., Fischl, B., Golland, P.: Spherical demons: Fast surface registration. In: Metaxas, D., Axel, L., Fichtinger, G., Székely, G. (eds.) MICCAI 2008, Part I. LNCS, vol. 5241, pp. 745-753. Springer, Heidelberg (2008)

17. Durrleman, S., Pennec, X., Trouvé, A., Ayache, N.: Sparse approximation of currents for statistics on curves and surfaces. In: Metaxas, D., Axel, L., Fichtinger, G., Székely, G. (eds.) MICCAI 2008, Part II. LNCS, vol. 5242, pp. 390-398. Springer, Heidelberg (2008) 